\title{
Magnetic Trapping of Metastable Calcium Atoms
}

\author{
Dirk P. Hansen * Janis R. Mohr, and Andreas Hemmerich \\ Institut für Laser-Physik, Universität Hamburg, Jungiusstrasse 9, D-20355 Hamburg, Germany
}

(Dated: October 30, 2018)

\begin{abstract}
Metastable calcium atoms, produced in a magneto-optic trap (MOT) operating within the singlet system, are continuously loaded into a magnetic trap formed by the magnetic quadrupole field of the MOT. At MOT temperatures of $3 \mathrm{mK}$ and $240 \mathrm{~ms}$ loading time we observe $1.1 \times 10^{8}$ magnetically trapped ${ }^{3} \mathrm{P}_{2}$ atoms at densities of $2.4 \times 10^{8} \mathrm{~cm}^{-3}$ and temperatures of $0.61 \mathrm{mK}$. In a modified scheme we first load a MOT for metastable atoms at a temperature of $0.18 \mathrm{mK}$ and subsequently release these atoms into the magnetic trap. In this case $240 \mathrm{~ms}$ of loading yields $2.4 \times 10^{8}$ trapped ${ }^{3} \mathrm{P}_{2}$ atoms at a peak density of $8.7 \times 10^{10} \mathrm{~cm}^{-3}$ and a temperature of $0.13 \mathrm{mK}$. The temperature decrease observed in the magnetic trap for both loading schemes can be explained only in part by trap size effects.
\end{abstract}

PACS numbers: 32.80.Pj, 42.50.Vk, 42.62.Fi, 42.50.-p

Earth alkaline atoms provide a unique combination of interesting spectroscopic features connected to their two valence electrons which give rise to singlet and triplet excitations. The singlet systems possess strong principle fluorescence lines well suited for laser cooling with remarkable efficiency. Yet, temperatures are limited to the $\mathrm{mK}$ domain, due to the absence of ground state Zeeman structure, a prerequisite for sub-Doppler techniques. The triplet systems, however, have readily accessible narrow band optical transitions that render possible refined laser cooling schemes with the promise of temperatures even beyond the microkelvin range. In fact, such schemes have recently been experimentally realized for strontium and calcium [1, 2, 3, 4]. Owing to their spectroscopic peculiarities such ultracold earth alkaline samples open up new prospects for ultraprecise atomic clocks [5, 6, 7] and cold collision studies which allow direct comparisons with ab initio theoretical calculations [8, 9, 10]. The formation of Bose-Einstein condensates (BEC, 11]) for this exciting group of atoms appears particularly desirable.

A key technique for obtaining BEC in alkalis and nobel gases has been magnetic trapping. This trapping technique outperforms optical techniques in two ways. It provides well controllable potential wells with sufficient steepness. The regime of high elastic collision rates, a precondition for efficient evaporative cooling, is thus easily accessible. Secondly, the presence of antibinding magnetic sublevels allows to actively force evaporation in a particular effective way by selectively expelling energetic atoms from the trap. The extension of this successful trapping technique to earth alkaline atoms may pave the route to $\mathrm{BEC}$ in this atom group. While the singlet ground state of these species lacks magnetic substructure, the ground state of the triplet system typically offers a particularly large Zeeman effect. Specifically, the long-lived ${ }^{3} \mathrm{P}_{2}, m_{J}=2$ state appears appropriate for mag-

*Electronic address: dhansen@physnet.uni-hamburg.de netic trapping and the formation of BEC. Recent calculations for calcium and strontium predict a positive scattering length (and thus stable BEC) for this state [10].

In this article we explore the application of magnetic trapping to the case of ${ }^{40} \mathrm{Ca}$. We demonstrate loading of a magnetic quadrupole trap with several times $10^{8}$ calcium atoms in the ${ }^{3} \mathrm{P}_{2}$ metastable state at peak densities near $10^{11} \mathrm{~cm}^{-3}$ and temperatures around $0.13 \mathrm{mK}$. We prepare cold Calcium ${ }^{3} \mathrm{P}_{2}$ atoms in the $\mathrm{mK}$ range at very high rates from a magneto-optic trap operating on the principle fluorescence line of the singlet system at $423 \mathrm{~nm}$ (called SMOT in the following) 12]. The excited state of the SMOT-transition has a small decay channel leading to the ${ }^{1} \mathrm{D}_{2}$ state $\left(\gamma_{1}=2180 \mathrm{~s}^{-1}[13]\right)$ and further on to the ${ }^{3} \mathrm{P}_{2}\left(\gamma_{2}=96 \mathrm{~s}^{-1}\right)$ and ${ }^{3} \mathrm{P}_{1}\left(\gamma_{3}=300 \mathrm{~s}^{-1}\right)$ triplet states (see Fig.1a for relevant Ca levels). While the atoms decaying via ${ }^{3} \mathrm{P}_{1}$ return to the ground state in about $3 \mathrm{~ms}$ and can be recycled into the SMOT, those decaying to ${ }^{3} \mathrm{P}_{2}$ represent a permanent loss that limits the SMOT life time to $21 \mathrm{~ms}$. Transfer rates into the ${ }^{3} \mathrm{P}_{2}$ state can exceed $10^{10}$ atoms/s at a temperature of about 2-3 $\mathrm{mK}$ determined by the Doppler temperature of the SMOT. In order to prepare even colder ${ }^{3} \mathrm{P}_{2}$ atoms, we superimpose a second magneto-optic trap (TMOT) for ${ }^{3} \mathrm{P}_{2}$ atoms using the narrow-band ${ }^{3} \mathrm{P}_{2} \rightarrow{ }^{3} \mathrm{D}_{3}$ triplet transition at $1978 \mathrm{~nm}$ [4]. If the TMOT is optimized for high loading rates it typically collects several times $10^{8}$ atoms within $240 \mathrm{~ms}$ at temperatures around $180 \mu \mathrm{K}$.

The magnetic quadrupole field shared by the SMOT and TMOT provides a natural test ground for magnetic trapping of ${ }^{3} \mathrm{P}_{2}$ atoms as has been recently discussed in detail in ref. 14]. The trapping potential is given by $U(x, y, z)=U_{0} \sqrt{\frac{1}{4}\left(x^{2}+y^{2}\right)+z^{2}}$ with $U_{0}=$ $2 \pi \hbar \times g \times m_{J} \times b \times(1.4 M H z /$ Gauss $)$, where $b$ is the magnetic field gradient, $m_{J}$ is the magnetic quantum number, and $g$ denotes the $\mathrm{g}$-factor. Due to the large value $\mathrm{g}=3 / 2$ for the ${ }^{3} \mathrm{P}_{2}$ state, for atoms in the low field seeking $m_{J}=2$ Zeeman component a magnetic field gradient $b$ of only 2.5 Gauss/cm is sufficient to compensate for gravity, and 10 Gauss/cm provide a significant trap 

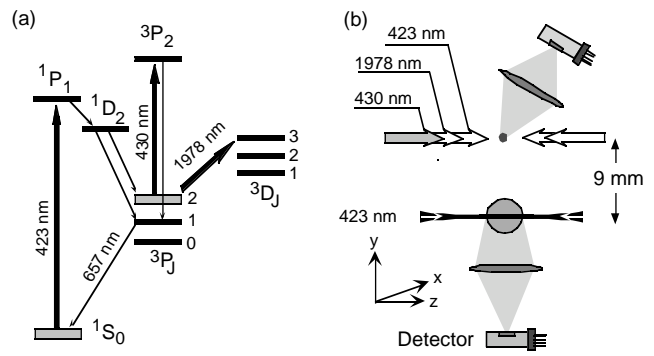

FIG. 1: (a) Relevant energy levels and transitions in ${ }^{40} \mathrm{Ca}$. (b) Sketch of the experimental setup. SMOT and TMOT beams are indicated only in the $\mathrm{z}$-direction.

potential of $2.5 \mathrm{mK} / \mathrm{cm}$.

The experimental setup is an extension of that used in ref. [4]. Fig.1b provides a sketch of the relevant elements. Three beams with $8 \mathrm{~mm}$ in diameter and $20 \mathrm{~mW}$ power, detuned from resonance by two times the natural line width, are retroreflected in order to form the SMOT. Atoms are provided from a Zeeman-decelerated atomic beam. By observation of the $423 \mathrm{~nm}$ fluorescence we measure $4 * 10^{7}$ atoms in the excited ${ }^{1} \mathrm{P}_{1}$ state of the SMOT. From the known transition rates (see e.g. ref. [13]) we can deduce a transfer rate into the ${ }^{3} \mathrm{P}_{2}$ state of $1.9 \times 10^{10}$ atoms/s. The magnetic quadrupole field with horizontal symmetry axis (z-axis) has a trap depth of $20.6 \mathrm{mK}$ and a magnetic field gradient (along the $\mathrm{z}^{-}$-axis) of $b=26 \mathrm{Gauss} / \mathrm{cm}$ in the origin. For monitoring the operation of the SMOT we record the fluorescence at $657 \mathrm{~nm}$ due to atoms decaying via ${ }^{1} \mathrm{D}_{2}$ and ${ }^{3} \mathrm{P}_{1}$ back to the ground state. In order to observe the ${ }^{3} \mathrm{P}_{2}$ atoms we optically pump them to the ${ }^{3} \mathrm{P}_{1}$ state by applying a $0.5 \mathrm{~ms}$ pulse of $430 \mathrm{~nm}$ light resonant with the ${ }^{3} \mathrm{P}_{2}(4 s 4 p) \rightarrow{ }^{3} \mathrm{P}_{2}(4 p 4 p)$ transition. The $1 / e^{2}$-radius of this optical pumping beam is $3 \mathrm{~mm}$. In order to work with a reduced optical pumping volume, a variable apperture placed inside this beam is imaged onto the atomic sample. The ${ }^{3} \mathrm{P}_{1}$ atoms decay to the ${ }^{1} \mathrm{~S}_{0}$ singlet ground state in $0.4 \mathrm{~ms}$ and are subject to ballistic expansion. Temperature measurements are performed with a time of flight method (TOF). A $10 \mathrm{~mm}$ wide and $0.5 \mathrm{~mm}$ thick sheet of light resonant with the ${ }^{1} \mathrm{~S}_{0} \rightarrow{ }^{1} \mathrm{P}_{1}$ transition is placed $9 \mathrm{~mm}$ below the center of the optical pumping beam (see Fig.1b). The fluorescence in this light sheet is recorded by a photo multiplier from below. Because we operate the TMOT with the same magnetic quadrupole field, the narrow bandwidth $(57 \mathrm{kHz})$ of the ${ }^{3} \mathrm{P}_{2} \rightarrow{ }^{3} \mathrm{D}_{3}$ transition is power-broadened to a peak value of $16 \mathrm{MHz}$, in order to obtain sufficient spatial capture volume. With $5 \mathrm{~mW}$ for each of the three retroreflected beams of 10 $\mathrm{mm}$ diameter the resonant peak saturation parameter is $7.6 \times 10^{4}$.

In Fig. 2 the operation of the magnetic trap is illustrated. Before $\mathrm{t}=0$, for about $240 \mathrm{~ms}$ atoms are Zeeman cooled, loaded into the SMOT, and transferred to

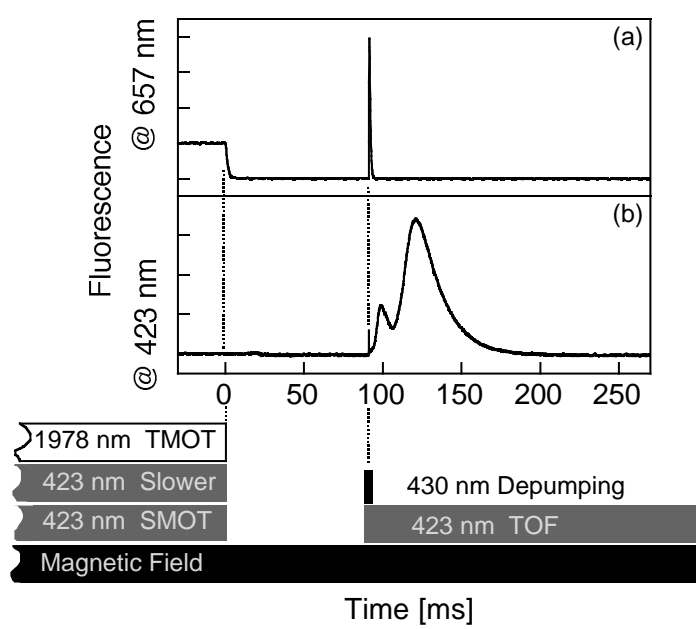

FIG. 2: Typical experimental sequence comprised of a $240 \mathrm{~ms}$ loading phase $(t<0)$, a variable phase of magnetic trapping, and a TOF phase initiated by a $0.5 \mathrm{~ms}$ optical pumping pulse at $430 \mathrm{~nm}$. The fluorescence at $657 \mathrm{~nm}$ (a) and a TOF signal (b) are shown.

the magnetically trapped ${ }^{3} \mathrm{P}_{2}$ state. At $\mathrm{t}=0$ the $423 \mathrm{~nm}$ beams are disabled, i.e. no further loading of the magnetic trap occurs which is seen from the drop of $657 \mathrm{~nm}$ fluorescence in trace (a). After a variable time delay the optical pumping pulse is applied which gives rise to a spike of $657 \mathrm{~nm}$ fluorescence, and a TOF spectrum is recorded (trace (b)). Alternatively, during the loading period before $t=0$ we may apply the TMOT. In this case the magnetic trap is not continuously loaded but at $\mathrm{t}=0$ the ${ }^{3} \mathrm{P}_{2}$ atoms trapped and cooled in the TMOT are suddenly released into the magnetic trap. Detection is performed as in the case of SMOT loading. While TMOT loading allows for lower initial temperatures, an attractive feature of SMOT loading is its continuous character, i.e. the possibility to add particles to the trap without disturbing those already trapped, as recently discussed in ref. 14] and demonstrated for chromium atoms in ref. [15].

By varying the trapping time in Fig.2 and observing the size of the $657 \mathrm{~nm}$ fluorescence peak in trace (a) we can measure the life time of the magnetic trap. The case of TMOT loading is shown in Fig.3a (open rectangles). For comparison, the filled rectangles show the decay of the TMOT itself. A surprisingly high transfer efficiency of about $75 \%$ is observed. The fitted exponentials (solid lines in Fig.3a) correspond to a model which neglects two-body collision losses. The decay time constants of $239 \pm 4 \mathrm{~ms}$ in the upper trace and $229 \pm 8 \mathrm{~ms} \mathrm{~ms}$ in the lower trace agree within the errors and are in accordance with the $10^{-8}$ mbar vacuum conditions. For SMOT loading the same decay time is found. Although a model accounting for inelastic two-body collisions yields slightly better fits for the lower trace in Fig.3a, slow fluctuations of the initial sample sizes in our present data do not allow us to extract reliable values for the collision rate. 

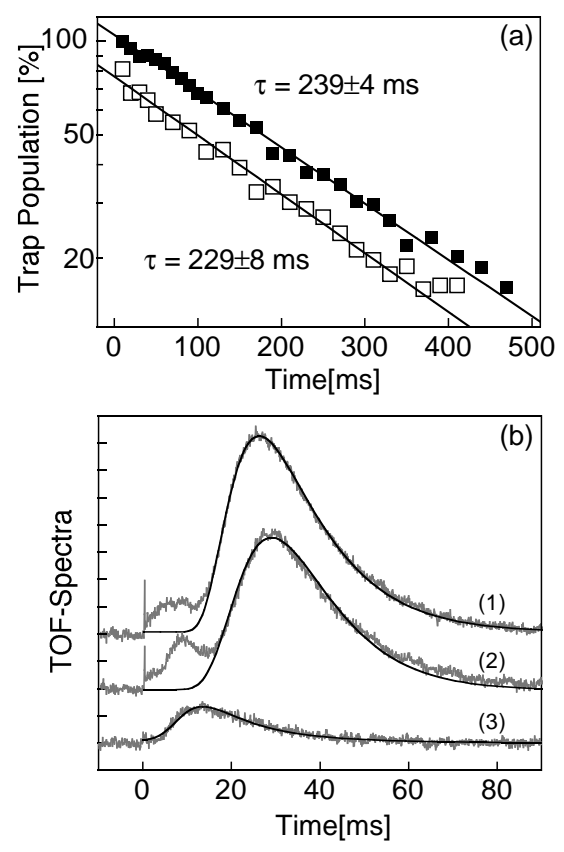

FIG. 3: (a) Life time measurement of the magnetic trap for TMOT loading (open rectangles) and the TMOT for comparison (filled rectangles). The solid lines show exponential fits. (b) TOF spectra (grey color) and corresponding theoretical fits (black lines) for the TMOT (1) and the magnetic trap for TMOT loading (2) and SMOT loading (3) respectively. All traces share the same scale.

In Fig.3b TOF spectra are shown for three different cases. The upper trace (1) shows a TOF spectrum for atoms trapped in the TMOT for $370 \mathrm{~ms}$ with a loading period of $240 \mathrm{~ms}$. In trace (2) the TMOT was loaded for $240 \mathrm{~ms}$ but subsequently the atoms were released into the magnetic trap and held there for $130 \mathrm{~ms}$. In trace (3) the SMOT continuously loaded the magnetic trap for $240 \mathrm{~ms}$ followed by $130 \mathrm{~ms}$ without further loading. Note that in either case (2) and (3) the temperature found in the magnetic trap is significantly lower than that of the loaded atomic sample. In trace (3) the initial sample in the SMOT has a temperature of about $3 \mathrm{mK}$, while that of the magnetically trapped sample is only $611 \mu \mathrm{K}$. In (2) the initial sample temperature is $182 \mu \mathrm{K}$ as seen in trace (1), while the temperature in the magnetic trap is only $134 \mu \mathrm{K}$. The fits in Fig.3b (solid black lines) used to evaluate temperatures are derived from a TOF model with three fit parameters: the initial vertical diameters and atom numbers of the fraction of atoms participating in the ballistic flight, and the temperatures. The initial sizes found in these fits are consistent with those observed. The diameter of the TMOT is estimated to be $2 \mathrm{~mm}$ by scanning the $430 \mathrm{~nm}$ optical pumping beam with $0.5 \mathrm{~mm}$ diameter through the atomic sample and observing the $657 \mathrm{~nm}$ fluorescence peak of Fig.2a. This corresponds to the best fit in trace (1) obtained for $2.4 \mathrm{~mm}$. The initial diameter for the TOF measurement of trace (2) is estimated similarly to be about $4 \mathrm{~mm}$ correspond- ing to the value of $4.1 \mathrm{~mm}$ in the best fit. The best fit value of the initial diameter for trace (3) of $7.9 \mathrm{~mm}$ does not reflect the size of the trapped population but is determined by the $3 \mathrm{~mm}$ maximal radius of the optical pumping beam. In traces (2) and (3) we also recognize a hot fraction of atoms occurring at early times in the TOF-spectra which are not accounted for in our model. Particularly, in the case of TMOT loading (trace (2)) a well distinguished hot fraction (at several $\mathrm{mK}$ ) is visible. For TMOT loading, TOF spectra recorded for trapping times shorter than $100 \mathrm{~ms}$ show additional structure which reflects non-equilibrium trap dynamics as is illustrated in Fig.4. The initial sharp peaks, resulting from the $430 \mathrm{~nm}$ depumping photons, indicate the release of the atoms from the magnetic trap. One recognizes an oscillation of population between hotter and colder fractions of atoms occuring at earlier or later times in the TOF spectra.

In order to explain the temperature decrease in the magnetic trap we first consider the case of SMOT loading in trace (3). The ${ }^{3} \mathrm{P}_{2}$ trap potential is continuously loaded by the cold flux of atoms emerging from the trapping volume of the SMOT with $\sigma=1 \mathrm{~mm} 1 / e^{2}$-radius via intermediate population of the ${ }^{1} \mathrm{D}_{2}$ state where the atoms spend on average $10 \mathrm{~ms}$. During this process the atoms remain subjected to magnetic trapping since the ${ }^{1} \mathrm{D}_{2}$ state itself provides a trap potential with $2 / 3$ of the size of that of the ${ }^{3} \mathrm{P}_{2}$ state. If the initial atomic sample is smaller than the equilibrium distribution inside the magnetic trap, an expansion occurs which reduces the mean kinetic energy of the initial sample $k_{B} T_{i}$ by the average potential energy of the final equilibrium distribution $\rho$ minus the average potential energy of the initial distribution. Thus, for the quadrupole potential $U$ the final kinetic energy is calculated to be $k_{B} T_{f}=k_{B} T_{i} / 3+$ $0.172 \times \sigma \times U_{0}$. The second term corresponds to $90 \mu \mathrm{K}$ for SMOT loading and may be neglected as compared to typical initial SMOT temperatures of $3 \mathrm{mK}$. Thus, temperatures above $1 \mathrm{mK}$ are expected, exceeding the observed $0.6 \mathrm{mK}$. In case of TMOT loading in trace (2) a similar deviation is found. Here $0.172 \times \sigma \times U_{0}$ amounts to $108 \mu \mathrm{K}$ and thus the expected temperature is $169 \mu \mathrm{K}$ as compared to $134 \mu \mathrm{K}$ observed.

Let us next estimate the capture efficiencies beginning with SMOT loading. We first calculate the average Zeeman detuning $\delta_{B}$ experienced by the different Zeeman components of the ${ }^{1} \mathrm{P}_{1}$ fraction of the SMOT sample in the quadrupole field $\mathrm{U}(\mathrm{x}, \mathrm{y}, \mathrm{z})$. With $b=26$ Gauss/cm we get $\delta_{B}\left(m_{J}\right) / \Gamma=0.08 \times m_{J}$. Using these Zeeman detunings we calculate the average relative excitation probabilities of the ${ }^{1} \mathrm{P}_{1}$ Zeeman components finding $37 \%$ for the high field seeking $m_{J}=-1$ state, $34 \%$ for the nonmagnetic state, and $29 \%$ for the low field seeking $m_{J}=1$ state. With the help of the Clebsch-Gordan coefficients we derive the relative populations of the Zeeman sublevels in the ${ }^{1} \mathrm{D}_{2}$ and ${ }^{3} \mathrm{P}_{2}$ states respectively. We find $18 \%$ population in the $m_{J}=2$, and $19 \%$ population in the $m_{J}=1$ low field seeking component of the ${ }^{3} \mathrm{P}_{2}$ level 


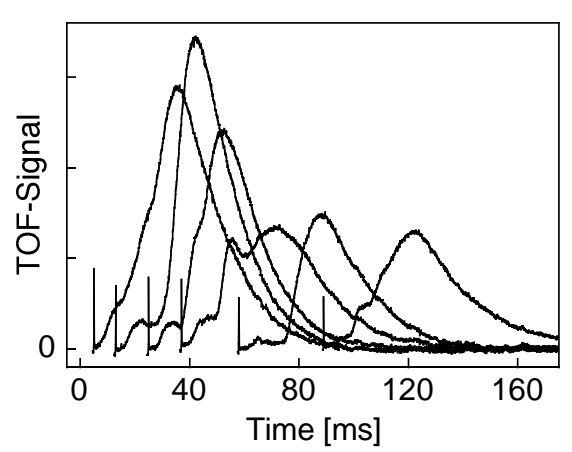

FIG. 4: TOF spectra for TMOT loading and short trapping durations (from left to right: $5 \mathrm{~ms}, 13 \mathrm{~ms}, 25 \mathrm{~ms}, 37 \mathrm{~ms}$, $58 \mathrm{~ms}, 89 \mathrm{~ms})$.

which are the two trapped states. In case of TMOT loading all atoms leaving the ${ }^{1} \mathrm{P}_{1}$ state should be captured in the TMOT and about $20 \%$ of those atoms should be transfered to each of the two magnetically trapped Zeeman sublevels. For SMOT loading only a fraction of the magnetically trapped atoms can contribute to the $657 \mathrm{~nm}$ fluorescence peak following the optical pumping pulse. In order to calculate the transfer efficiency by optical pumping we have numerically integrated the product of the thermal equilibrium distribution in the ${ }^{3} \mathrm{P}_{2}$ potential $\rho(x, y, z)=(32 \pi)^{-1}\left(U_{0} / k_{B} T\right)^{3} \exp \left(-U(x, y, z) / k_{B} T\right)$ and the local optical pumping probability during the $0.5 \mathrm{~ms}$ pumping pulse. To derive the local pumping probability we have solved a rate equation including all levels involved and account for the local intensity, polarization and the Zeeman detunings from resonance due to the magnetic quadrupole field. Temperatures are inserted as obtained from Fig.3b. For SMOT loading we find that a fraction of $39 \%$ of the $m_{J}=2$ atoms and $14 \%$ of the $m_{J}=1$ atoms is transfered. For TMOT loading no loss should occur in the optical pumping transfer.

For SMOT loading we typically observe a $657 \mathrm{~nm}$ fluorescence peak with $2.9 \times 10^{7}$ atoms. Accounting for the expected transfer efficiency by optical pumping discussed in the previous paragraph we obtain $5.4 \times 10^{7}$ trapped $m_{J}=2$ atoms and $5.7 \times 10^{7}$ trapped $m_{J}=1$ atoms. Assuming thermal equilibrium at $611 \mu \mathrm{K}$, the peak density is $3.4 \times 10^{8} \mathrm{~cm}^{-3}$ for $m_{J}=2$ atoms and $1 \times 10^{8} \mathrm{~cm}^{-3}$ for $m_{J}=1$ atoms. Accounting for the loading time of $240 \mathrm{~ms}$ a capture rate of the $m_{J}=2$ component of $3.6 \times 10^{8} \mathrm{~s}^{-1}$ is observed. This is to be compared with $18 \%$ of the overall transfer rate of $1.9 \times 10^{10} \mathrm{~s}^{-1}$, i.e. $3.4 \times 10^{9} \mathrm{~s}^{-1}$. For TMOT loading we find $2.4 \times 10^{8}$ fluorescing atoms, and accordingly $1.2 \times 10^{8}$ atoms in each of the two magnetically trapped Zeeman sublevels. Assuming thermal equilibrium at $135 \mu \mathrm{K}$, we find a peak density of $6.7 \times$ $10^{10} \mathrm{~cm}^{-3}$ for $m_{J}=2$ atoms and $2 \times 10^{10} \mathrm{~cm}^{-3}$ for $m_{J}=1$ atoms. The observed $m_{J}=2$ capture rate is $7.9 \times 10^{8} \mathrm{~s}^{-1}$ and has to be compared with $20 \%$ of the overall transfer rate of $1.9 \times 10^{10} s^{-1}$, i.e. $3.8 \times 10^{9} s^{-1}$. Presently, we cannot resolve the discrepancies between the expected and the observed capture rates which amount to a factor 9.4 for SMOT loading and a factor 4.8 for TMOT loading.

In summary, we have applied magnetic trapping to the group of earth alkaline atoms, preparing several times $10^{8}$ calcium atoms in the ${ }^{3} \mathrm{P}_{2}$ metastable state at peak densities near $10^{11} \mathrm{~cm}^{-3}$ and temperatures around $0.13 \mathrm{mK}$. This represents favorable starting conditions for the formation of a metastable calcium BEC. Technical improvements (e.g. an extra transient cooling phase in the TMOT scheme, as explained in ref. [4], or a simple decrease of the background pressure) promise at least an order of magnitude improvement of the initial phase space density.

\section{Acknowledgments}

This work has been supported in part by the Deutsche Forschungsgemeinschaft (SPP 1116), DAAD probral/bu, and the European training network CAUAC.
[1] H. Katori, T. Ido, Y. Isoya, and M. Kuwata-Gonokami, Phys. Rev. Lett. 82, 1116-1119 (1999).

[2] T. Binnewies et al., Phys. Rev. Lett. 87, 123002 (2001).

[3] E. A. Curtis, C. W. Oates, and L. Hollberg, Phys. Rev. A 64, 031403(R) (2001).

[4] J. Grünert, and A. Hemmerich, Phys. Rev. A 65, 041401(R) (2002).

[5] T. Kisters, K. Zeiske, F. Riehle, and J. Helmcke, J. Appl. Phys. B 59, 89 (1994).

[6] F. Ruschewitz et al., Phys. Rev. Lett. 80, 3173 (1998).

[7] C. Oates, F. Bondu, R. Fox, and L. Hollberg, Eur. Phys. J. D. 7, 449 (1999).

[8] T. P. Dinneen, K. R. Vogel, E. Arimondo, J. L. Hall, and A. Gallagher, Phys. Rev. A 59, 1216 (1999).
[9] M. Machholm, P. S. Julienne, and K.-A. Suominen, Phys. Rev. A 64, (2001).

[10] A. Derevianko et al., http://xxx.lanl.gov/abs/physics/ 0210076 (2002).

[11] M. H. Anderson et al., Science 269, 198 (1995).

[12] J. Grünert, G. Quehl, V. Elman, and A. Hemmerich, J. Mod. Opt.47, 2733 (2000); J. Grünert and A. Hemmerich, J. Appl. Phys. B 73, 1-4 (2000).

[13] N. Beverini, F. Giammanco, E. Maccioni, F. Strumia, and G. Vissani, J. Opt. Soc. Am. B 6, 2188-2193 (1989).

[14] T. Loftus, J. Bochinski, and T. Mossberg, Phys. Rev. A 66, 013411 (2002).

[15] J. Stuhler et al., Phys. Rev. A 64, 031405 (2001). 